\title{
High-NA aberration retrieval with the Extended Nijboer-Zernike vector diffraction theory: Erratum
}

\section{S. van Haver \\ s.vanhaver@tudelft.nl}

\section{J.J.M. Braat}

j.j.m.braat@tudelft.nl

\section{P. Dirksen}

peter.dirksen@philips.com

A.J.E.M. Janssen

a.j.e.m.janssen@philips.com
Optics Research Group, Faculty of Applied Sciences, Delft University of Technology, Lorentzweg 1, NL-2628 CJ Delft, The Netherlands

Optics Research Group, Faculty of Applied Sciences, Delft University of Technology, Lorentzweg 1, NL-2628 CJ Delft, The Netherlands

Philips Research Europe, Professor Holstlaan 4, NL-5656 AA Eindhoven, The Netherlands

\section{[DOI: 10.2971/jeos.2007.07011e]}

In the paper "High-NA aberration retrieval with the Extended Nijboer-Zernike vector diffraction theory" by S. van Haver, J.J.M. Braat, P. Dirksen and A.J.E.M. Janssen, published in J. Europ. Opt. Soc. Rap. Public. 1, 06004 (2006), some regrettable notation errors are present in Eq.(10), page 06004-3. The expression gives the azimuthal Fourier components $\Psi_{a n}^{m}(r, f)$ of the analytically calculated intensity distribution in the focal region of a high-numerical-aperture focused beam as a function of the radial and axial coordinates, $r$ and $f$, respectively. On line 4 of Eq.(10) in this publication, the upper index of the coefficient $\beta$ was erroneously given as $m$ but should have been $-m$. On line 10 of the same equation, the lower indices of $\Psi_{v ; 0,2}^{(m+2) *}$ should be changed into $\Psi_{v ; 2,0}^{(m+2) *}$; on line 14, the lower indices of $\Psi_{v ; 0,2}^{(-m+2)}$ should become $\Psi_{v ; 2,0}^{(-m+2)}$. The correct version of Eq.(10) for $\Psi_{a n}^{m}(r, f)$ is reproduced in full below,

$$
\begin{aligned}
& \Psi_{a n}^{m}(r, f)=\frac{\beta_{0}^{0}}{2} \sum_{v}\left\{\beta _ { v } ^ { m * } ( 2 - \epsilon _ { v , m } ) \left[\Psi_{v ; 0,0}^{m *}+s_{0}^{2} \times\right.\right. \\
& \left\{\left(\Psi_{v ; 1,1}^{m *}+\Psi_{v ;-1,-1}^{m *}\right)+\frac{s_{0}^{2}}{2}\left(\Psi_{v ; 2,2}^{m *}+\Psi_{v ;-2,-2}^{m *}\right)-2 \operatorname{Im}\left(a b^{*}\right) \times\right. \\
& \left.\left.\left[\left(\Psi_{v ; 1,1}^{m *}-\Psi_{v ;-1,-1}^{m *}\right)+\frac{s_{0}^{2}}{2}\left(\Psi_{v ; 2,2}^{m *}-\Psi_{v ;-2,-2}^{m *}\right)\right]\right\}\right] \\
& +\beta_{v}^{-m}\left(2-\epsilon_{v, m}\right)\left[\Psi_{v ; 0,0}^{-m}+s_{0}^{2}\left\{\left(\Psi_{v ; 1,1}^{-m}+\Psi_{v ;-1,-1}^{-m}\right)\right.\right. \\
& +\frac{s_{0}^{2}}{2}\left(\Psi_{v ; 2,2}^{-m}+\Psi_{v ;-2,-2}^{-m}\right)-2 \operatorname{Im}\left(a b^{*}\right) \times
\end{aligned}
$$

$$
\begin{aligned}
& \left.\left.\left[\left(\Psi_{v ; 1,1}^{-m}-\Psi_{v ;-1,-1}^{-m}\right)+\frac{s_{0}^{2}}{2}\left(\Psi_{v ; 2,2}^{-m}-\Psi_{v ;-2,-2}^{-m}\right)\right]\right\}\right] \\
& +\beta_{v}^{(m-2) *}\left[\left(|a|^{2}-|b|^{2}\right)+2 i \operatorname{Re}\left(a b^{*}\right)\right] \times \\
& s_{0}^{2}\left\{\Psi_{v ; 0,2}^{(m-2) *}+\left(1-\epsilon_{v, m-2}\right)\left[\Psi_{v ;-2,0}^{(m-2) *}-2 \Psi_{v ;-1,+1}^{(m-2) *}\right]\right\} \\
& +\beta_{v}^{(m+2) *}\left[\left(|a|^{2}-|b|^{2}\right)-2 i \operatorname{Re}\left(a b^{*}\right)\right] \times \\
& s_{0}^{2}\left\{\left(1-\epsilon_{v, m+2}\right) \Psi_{v ; 2,0}^{(m+2) *}+\Psi_{v ; 0,-2}^{(m+2) *}-2 \Psi_{v ;+1,-1}^{(m+2) *}\right\} \\
& +\beta_{v}^{(-m-2)}\left[\left(|a|^{2}-|b|^{2}\right)-2 i \operatorname{Re}\left(a b^{*}\right)\right] \times \\
& s_{0}^{2}\left\{\Psi_{v ; 0,2}^{(-m-2)}+\left(1-\epsilon_{v, m+2}\right)\left[\Psi_{v ;-2,0}^{(-m-2)}-2 \Psi_{v ;-1,+1}^{(-m-2)}\right]\right\} \\
& +\beta_{v}^{(-m+2)}\left[\left(|a|^{2}-|b|^{2}\right)+2 i \operatorname{Re}\left(a b^{*}\right)\right] \times \\
& \left.s_{0}^{2}\left\{\left(1-\epsilon_{v, m-2}\right) \Psi_{v ; 2,0}^{(-m+2)}+\Psi_{v ; 0,-2}^{(-m+2)}-2 \Psi_{v ;+1,-1}^{(-m+2)}\right\}\right\} .
\end{aligned}
$$

The numerical results described in the paper were not affected by the notational errors in Eq.(10) as it was shown in Figure 5 of the paper by the perfect convergence to their original values of the retrieved $\beta$-coefficients, down to machine precision. The errors in question were not present in the computational program that was used for the retrieval of the coefficients. 